\title{
EVALUACIÓN DE UN SISTEMA DE FOTOCATÁLISIS HETEROGÉNEA Y PASTEURIZACIÓN PARA DESINFECCIÓN DE AGUAS LLUVIAS
}

\author{
EVALUATION OF A HETEROGENEOUS PHOTOCATALYSIS \\ AND PASTEURIZATION SYSTEM FOR RAINWATER DISINFECTION
}

Angie Catherin Quintero Agudelo*

Camilo Andrés Vargas Terranova**

Juan Pablo Sanabria Alcantar ${ }^{* *}$

Fecha de recepción: 21 de octubre de 2016

Fecha de revisión: 9 de abril de 2017

Fecha de aprobación: 4 de julio de 2017

Cómo citar: A. C. Quintero Agudelo, C. A. Vargas Terranova y J. P. Sanabria Alcantar, "Evaluación de un sistema de fotocatálisis heterogénea y pasteurización para desinfección de aguas Iluvias," Ciencia e Ingeniería Neogranadina, vol. 28 , no. 1, pp. 117-134. DOI: http://dx.doi.org/10.18359/rcin.2350

\section{RESUMEN}

La fotocatálisis heterogénea y la pasteurización para la desinfección del agua se presentan como una alternativa de potencial implementación y operación, porque buscan el aprovechamiento de la radiación y la temperatura, y la hacen apta para emplearla en múltiples locaciones, principalmente donde existan fuentes de abastecimiento que presenten bajos niveles de turbiedad. Para verificar la efectividad de estas técnicas, se evaluó una unidad compuesta por un sistema de captación de aguas lluvias, un filtro lento de arena y un sistema de desinfección solar compuesto por fotocatálisis heterogénea, que emplea como catalizador dióxido de titanio $\left(\mathrm{TiO}_{2}\right)$ en forma de

Ingeniera Ambiental y Sanitaria, Vigilancia Sanitaria. Trabaja en Subred Integrada de Servicios de Salud Centro Oriente E.S.E., Bogotá, Colombia. Correo electrónico: qangie32@unisalle.edu.co. ORCID: http://orcid. org/0000-0002-9113-5685

** Ingeniero Ambiental y Sanitario, magíster consultor ambiental, Quipus S.A., y profesor, Universidad de La Salle, Bogotá, Colombia. Correo electrónico: canvargas@unisalle.edu.co. ORCID: http://orcid.org/0000-00021926-7026

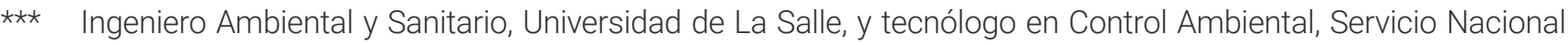
de Aprendizaje (SENA). Bogotá, Colombia. Correo electrónico: sjuan25@unisalle.edu.co. ORCID: https://orcid. org/0000-0003-3309-1300
} 
anatasa 99 \% en un lecho fijo, y pasteurización con un proceso de recirculación, en una comunidad ubicada en la zona sur de Bogotá. La caracterización del afluente mostró valores de turbiedad iguales a 3,29 UNT, pH de 6,4, alcalinidad de 2,5 $\mathrm{mg} \mathrm{CaCO}_{3} \mathrm{~L}^{-1}$ dureza de 0,65 $\mathrm{mg} \mathrm{CaCO}_{3} \mathrm{~L}^{-1} \mathrm{y}$ presencia de coliformes identificados en placas de petrifilm. La eliminación de microorganismos se hizo efectiva en días con índices de radiación por encima de 4UV (longitudes de onda de 315$400 \mathrm{~nm}$ ), estados del tiempo soleados o parcialmente nublados y valores de turbiedad de 4 UNT para cargas hidráulicas por debajo de $60 \mathrm{~m}^{3} \mathrm{~m}^{-2}-\mathrm{d}^{-1}$. El efluente presentó efectividad próxima al $60 \%$ en la remoción de turbiedad y $100 \%$ en coliformes. Los resultados obtenidos permiten el uso del agua tratada en actividades complementarias, excluyendo el consumo humano.

Palabras clave: desinfección, fotocatálisis heterogénea, pasteurización, calidad del agua.

\section{ABSTRACT}

Heterogeneous photocatalysis and pasteurization for water disinfection, are presented as an alternative to potential implementation and operation, because they seek the use of radiation and temperature making it suitable for use in multiple locations, principally where there are sources of supply that low levels of turbidity. To verify the effectiveness of these techniques, we evaluated a composite unit of a rainwater harvesting system, a slow sand filter and a solar disinfection system composed of heterogeneous photocatalysis, which uses as a catalyst of titanium dioxide (TiO2) in the form of anatase $99 \%$ in a fixed bed, and pasteurization with a recirculation process, in a community located in the South zone of Bogota. The characterization of the tributary showed turbidity values equal to $3.29 \mathrm{NTU}, \mathrm{pH} 6.4$, alkalinity $\left(\mathrm{CaCO}_{3}\right) 2.5$ $\mathrm{mg} \mathrm{L}^{-1}$ hardness of $0.65 \mathrm{mg} \mathrm{CaCO}_{3} \mathrm{~L}^{-1}$ and presence of coliforms identified in petrifilm plates. Elimination of microorganisms became valid on days with indices of radiation above 4UV (wavelengths of $315-400 \mathrm{~nm}$ ), sunny or partially cloudy weather and values of Turbidity 4 NTU to hydraulic loads below $60 \mathrm{~m}^{3} \mathrm{~m}^{-2} \mathrm{~d}^{-1}$. The effluent presented to the $60 \%$ effectiveness in the removal of turbidity and coliform $100 \%$. The results obtained allow the use of the treated water in complementary activities excluding the human consumption.

Keywords: Disinfection, heterogeneous photocatalysis, pasteurization, water quality.

\section{INTRODUCCION}

La desinfección es un proceso importante dentro de un sistema de tratamiento de agua, independientemente de su fuente. Se busca a partir de ella la eliminación de microorganismos existentes que puedan causar enfermedades [1]. Estudios reali- zados por la Organización Mundial de la Salud (OMS) han detectado altos niveles de coliformes totales y coliformes fecales en agua lluvia [2], lo que evidencia la necesidad de implementar procesos de tratamiento que sirvan como una alternativa de desinfección. 
La fotocatálisis heterogénea, es una alternativa de desinfección solar, a partir de la absorción de longitudes de onda inferiores a $400 \mathrm{~nm}$ [3] y [4] en presencia de un catalizador [5]. La luz y el catalizador son necesarios para alcanzar o acelerar una reacción química con el objeto de formar radicales hidroxilo [6]-[9]. Este mecanismo de desinfección se basa en un fenómeno físico por el cual las ondas cortas de la radiación ultravioleta [3] producen una división entre la pared de la célula y la membrana de los microorganismos y los virus, lo cual deteriora su membrana celular e inhibe su reproducción [10], y esto genera su eliminación sin causar cambios fisicoquímicos que puedan alterar el agua tratada [3]

El catalizador más ampliamente usado es el dióxido de titanio $\left(\mathrm{TiO}_{2}\right)$, en forma de anatasa un $99 \%$ y en forma de rutilo un $1 \%$ [6]-[11]. El dióxido de titanio $\left(\mathrm{TiO}_{2}\right)$ no es tóxico ni genera subproductos cancerígenos [7] y entre sus propiedades ópticas se encuentra como una substancia con un índice de refracción alto, lo que permite reflejar la luz UV [4]. Fisicoquímicamente las aguas tratadas por fotocatálisis heterogénea incluyen de forma especial $\mathrm{pH}$ a diferentes rangos [5]-[12], variaciones de temperatura sin afectar significativamente la velocidad de las reacciones [10] y turbiedades no mayores a 5 UNT [3]. El ion inhibidor de mayor influencia es el fosfato, puesto que impide la adsorción de aminoácidos sobre las partículas de $\mathrm{TiO}_{2}$, los carbonatos y otras especies que pueden reaccionar con los radicales hidroxilo; de esta manera, compite con los microorganismos y reduce la eficiencia del fotocatalizador [13].
El uso de reactores de lecho fijo es común para reacciones catalíticas, ya que pone en contacto el catalizador con el reactante para que la reacción se produzca de manera idónea. En estos, las partículas dispuestas en un lecho a través del cual el fluido circula están inmovilizadas y, por tanto, en íntimo contacto unas con otras [14].

Otra de las múltiples aplicaciones de la desinfección solar es la pasteurización, conocida como Solar Water Disinfection (SODIS) [3]-[15], la cual se basa en un proceso térmico que consiste en elevar la temperatura del agua por un tiempo suficiente en contenedores preferiblemente de color negro, con lo cual se logra la absorción y conservación del calor proveniente de la radiación solar [3]. Se ha comprobado que un incremento de la temperatura tiene gran incidencia sobre todos los microorganismos; en general, la mayoría de las bacterias mueren aproximadamente a $65^{\circ} \mathrm{C}$ en tiempo determinado de 30 minutos [3].

La participación de la luz solar en la eliminación de productos químicos de síntesis del ambiente está bien documentada en los muchos artículos publicados en los últimos años. [16]. Algunas investigaciones apuntan al tratamiento de aguas residuales industriales especialmente, y obtienen reducciones entre 6 y $46 \%$ de la demanda química de oxigeno (DQO), la cual hace referencia a la cantidad de sustancias susceptibles a la oxidación de compuestos químicos presentes en el agua [17] y carbono orgánico total (COT), que determina la cantidad de carbono representativo dentro de un compuesto orgánico con porcentajes de degradación cercanos al $98 \%$ [11]. 
La fotocatálisis es una tecnología que podría aportar soluciones innovadoras para la desinfección del agua. Así, por ejemplo, la aplicación de un sistema fotocatalítico después de un tratamiento físico como la filtración representa un acople con gran potencial para eliminar los microorganismos presentes en el agua [18].

En estimaciones sobre las concentraciones de SODIS con dióxido de titanio $\left(\mathrm{TiO}_{2}\right)$ correctas, se calcula que no existe diferencia significativa con valores entre $0,288,0,5011$ y $1,0073 \mathrm{mg} / \mathrm{ml}$, puesto que hay eliminación de coliformes en cualquiera de los casos [13]. Sin embargo, diferentes autores atribuyen otras concentraciones mayores entre rangos como $2 \mathrm{~g} \mathrm{~L}^{-1}$ y $1 \mathrm{~g} \mathrm{~L}^{-1}$ en un reactor con lámparas de agitación y en $100 \mathrm{mg} \mathrm{L}^{-1}$ en reactores con radiación solar [15]. Es decir, las concentraciones varían dependiendo de la técnica empleada.

En el método SODIS convencional, la eliminación de microorganismos se realiza en más de una hora, mientras que en presencia del catalizador tarda tan solo unos pocos minutos [13]-[15]. Estos métodos de desinfección en general son mayormente empleados en zonas rurales por su bajo costo y su fácil implementación [19]. Empero, factores como la geografía, el estado del tiempo, el número de horas de exposición, el volumen y el material de los envases que contienen el agua, la turbiedad y el color son, entre otros, los parámetros que podrían interferir en una desinfección oportuna [3].

Las regiones más favorables para aplicar SODIS se ubican entre las latitudes $15^{\circ} \mathrm{N}$ y $35^{\circ} \mathrm{N}$ (así como $15^{\circ} \mathrm{S}$ y $35^{\circ} \mathrm{S}$ ), la turbiedad del agua no debe exceder los 30 UNT, Ios recipientes empleados son botellas de polietileno de tereftalato (PET) transparentes de hasta $2 \mathrm{~L} y$ se deben exponer de 5 a 6 horas bajo un cielo soleado o ligeramente nublado desde las 9 a. $\mathrm{m}$. hasta las $3 \mathrm{p}$. m., puesto que, cumpliendo estas condiciones, la eficacia de SODIS para la pasteurización es del 99,9\% [20]. No obstante, Castillo, López y Bandala [21] pronostican que la comunidad científica internacional continuará observando el desarrollo de la inversión en esta área, puesto que aún resulta insuficiente la investigación.

Lo anterior sirvió de base para diseñar, construir y evaluar un sistema de desinfección solar de agua con bajos niveles de turbiedad a partir de fotocatálisis heterogénea y pasteurización, para agua lluvia.

La determinación de microorganismos presentes en el agua, más específicamente coliformes totales o fecales, se realizó por medio de placas petrifilm, las cuales dan resultados confirmados entre 24 a 48 horas [22]

\section{MATERIALES Y MÉTODOS}

\subsection{Fuente de captación}

La fuente de abastecimiento del sistema de desinfección solar correspondió al agua lluvia. Para su tratamiento, se adecuaron instalaciones de una vivienda para su captura y conducción. Se caracterizaron como parámetros in situ turbiedad, $\mathrm{pH}$, temperatura, conductividad y solidos disueltos. El estudio se realizó entre las coordenadas geográficas $4^{\circ} 16^{\prime} 57^{\prime \prime}$ y $4^{\circ} 23^{\prime} 28^{\prime \prime}$ de latitud Norte y $74^{\circ} 10^{\prime} 57^{\prime \prime}$ y $74^{\circ} 13^{\prime} 23^{\prime \prime}$ longitud Oeste, según el meridiano de Greenwich. 
También se valoraron, como se muestra en la Tabla 1, los parámetros acidez, alcalinidad, dureza, aluminio, hierro, nitritos, nitratos, fosfatos, fluoruros y solidos suspendidos totales; a partir de muestreos puntuales, en respuesta a la resolución 2115 de 2007, ${ }^{1}$ y condiciones idóneas de preservación, custodia y transporte de muestras.

\subsection{Diseño y construcción del sistema} Las unidades de tratamiento consideradas para el sistema propuesto se exponen en la Fig. 1

Tabla 1. Caracterización inicial del agua lluvia en la zona de estudio

\begin{tabular}{|l|l|l|}
\hline \multicolumn{1}{|c|}{ Parámetro } & \multicolumn{1}{c|}{ Unidad } & \multicolumn{1}{c|}{ Valor } \\
\hline $\mathrm{pH}$ & Unidades & 6,4 \\
\hline Temperatura & $\left({ }^{\circ} \mathrm{C}\right)$ & 16,54 \\
\hline Conductividad & $\left(\mathrm{ms}^{\mathrm{cm}}\right)$ & 0,08 \\
\hline Solidos Disueltos & $(\mathrm{ppm})$ & 0,04 \\
\hline Turbiedad & $(\mathrm{UNT})$ & 3,29 \\
\hline Acidez & $\left(\mathrm{mg} \mathrm{L}^{-1}\right)$ & 2 \\
\hline Alcalinidad & $\left(\mathrm{mg} \mathrm{CaCO3} \mathrm{L}^{-1}\right)$ & 2,5 \\
\hline Dureza & $\left({\left.\mathrm{mg} \mathrm{CaCO} ~ \mathrm{~L}^{-1}\right)}^{-1}\right.$ & 0,65 \\
\hline Nitratos & $\left(\mathrm{mg} \mathrm{L}^{-1}\right)$ & 0,4 \\
\hline Nitritos & $\left(\mathrm{mg} \mathrm{L}^{-1}\right)$ & 0,1 \\
\hline Fosfatos & $\left(\mathrm{mg} \mathrm{L}^{-1}\right)$ & 0,85 \\
\hline Aluminio & $\left(\mathrm{mg} \mathrm{L}^{-1}\right)$ & 0,025 \\
\hline Fluoruros & $\left(\mathrm{mg} \mathrm{L}^{-1}\right)$ & 0,55 \\
\hline Cloruros & $\left(\mathrm{mg} \mathrm{L}^{-1}\right)$ & 0,2 \\
\hline
\end{tabular}

Fuente: elaboración propia.

1 Resolución 2115 de 2007 del Ministerio de la Protección Social y Ministerio de Ambiente, Vivienda y DesarroIlo Territorial, por medio de la cual se señalan características, instrumentos básicos y frecuencias del sistema de control y vigilancia para la calidad del agua para consumo humano. 


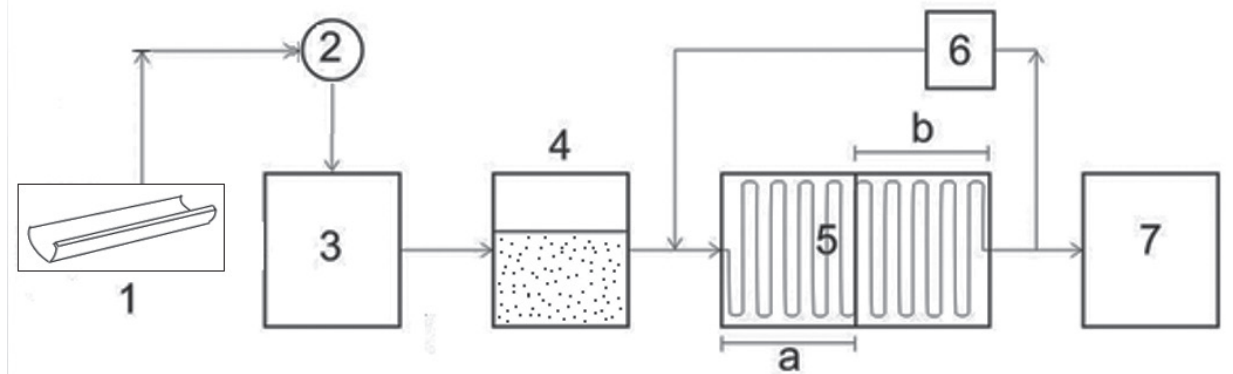

Fig. 1. Diagrama de flujo del sistema: 1) canales de captación de agua lluvia; 2) tanque interceptor de primeras aguas; 3 ) tanque de almacenamiento de agua lluvia; 4) filtro lento de lechos áridos; 5) sistema de desinfección solar, a) fotocatálisis heterogénea y b) pasteurización; 6) sistema de recirculación; 7) tanque de distribución final.

Fuente: elaboración propia.

\subsubsection{Captación de aguas lluvia}

Para estimar las necesidades de captación y almacenamiento del efluente, primero se calcularon los valores de precipitación promedio completando los registros faltantes por el método aritmético [23], con base en información de las estaciones meteorológicas y pluviométricas más cercanas a la zona, pertenecientes al Instituto de Hidrología, Meteorología y Estudios Ambientales de Colombia (Ideam).

El proceso de cálculo y diseño respondió a la metodología definida por el Centro Pa- namericano de Ingeniería Sanitaria y Ciencias del Ambiente (Cepis) [24]. Con esto se obtuvo el volumen del tanque de almacenamiento, un tanque interceptor de primeras aguas y el porcentaje de la demanda mensual por abastecer.

El tejado que recoge el agua lluvia es de material zinc; las canales, de aluminio; los tanques de almacenamiento e interceptor son comerciales y de plástico. La tubería del sistema es totalmente de PVC con diámetros de $3 / 4^{\prime \prime}$ y $1 / 2$ " (ver Fig. 2).

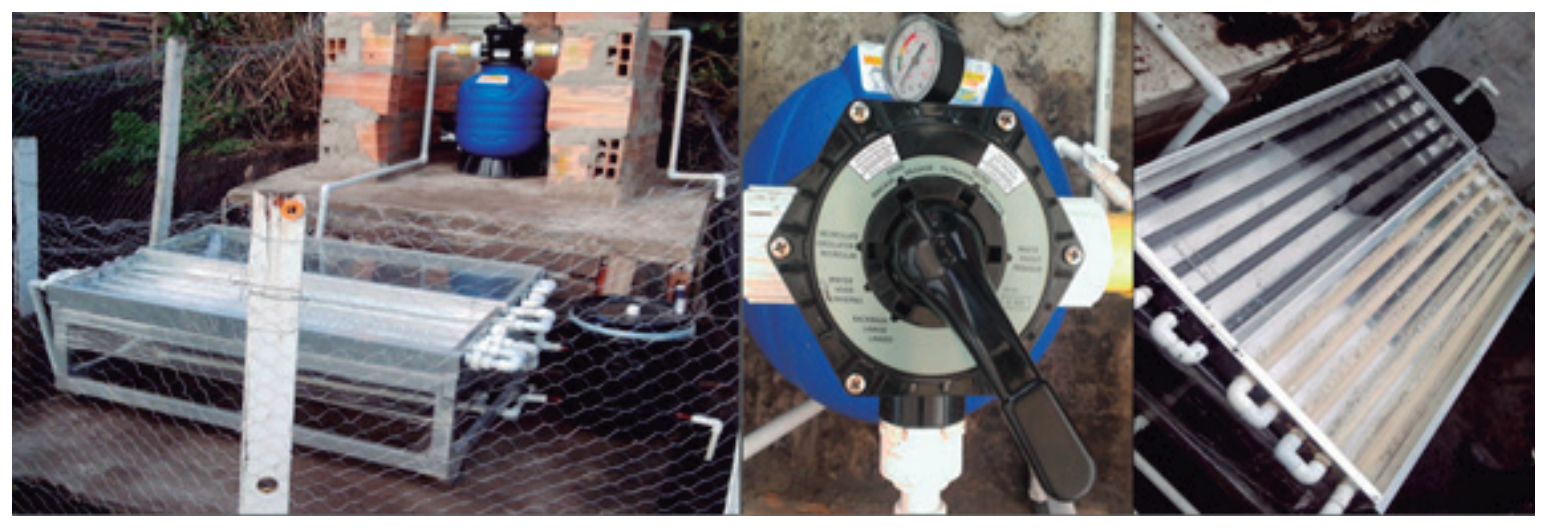

Fig. 2 Collage visualización del tratamiento

Fuente: elaboración propia. 


\subsubsection{Filtro lento de lechos áridos}

Entendiendo las necesidades para el tratamiento asociadas a niveles bajos de turbiedad, se diseñó un filtro con lechos áridos de grava fina $(3 \mathrm{~mm})$ y arena $(1 \mathrm{~mm})$ que retienen partículas [25], y se compró a la empresa FiltraH2o Ltda. La capacidad instalada del filtro se determinó aforando el caudal máximo de operación, por el método volumétrico [26] y fue regulado por medio de una válvula de bola de $1 / 2$ ".

\subsubsection{Desinfección solar por fotocatálisis heterogénea y pasteurización}

Para la construcción e implementación de las unidades de fotocatálisis heterogénea y pasteurización se definieron su geometría y óptica [27]. La geometría respondió a una inclinación de $5^{\circ}$ en dirección Oriente-Occiden- te, teniendo en cuenta la posición del Sol y la latitud en la zona, y así favorecer la radiación efectiva sobre la unidad en dicha dirección. En investigaciones para sistemas que emplean $\mathrm{TiO}_{2}$ en suspensión, la sedimentación del catalizador a lo largo del circuito hidráulico debe evitarse, por lo que se recomiendan diámetros entre 25 y $50 \mathrm{~mm}$ [28]. Sin embargo, en este caso el diámetro [27] se fijó experimentalmente en $20 \mathrm{~mm}$ para evitar problemas con la absorbancia del $\mathrm{TiO}_{2}$, que impiden que la radiación llegue completa a la parte interna del tubo, puesto que se planteó un sistema de lecho fijo.

La óptica se manejó a partir de la implementación de vidrio tipo Pyrex, con buenas garantías de transmitancia (Fig. 3) e importante captura de rayos UV; así, se mantuvieron las necesidades para la reacción fotocatalítica para radiación cuya longitud de onda sea menor a $400 \mathrm{~nm}$ [3], [4].

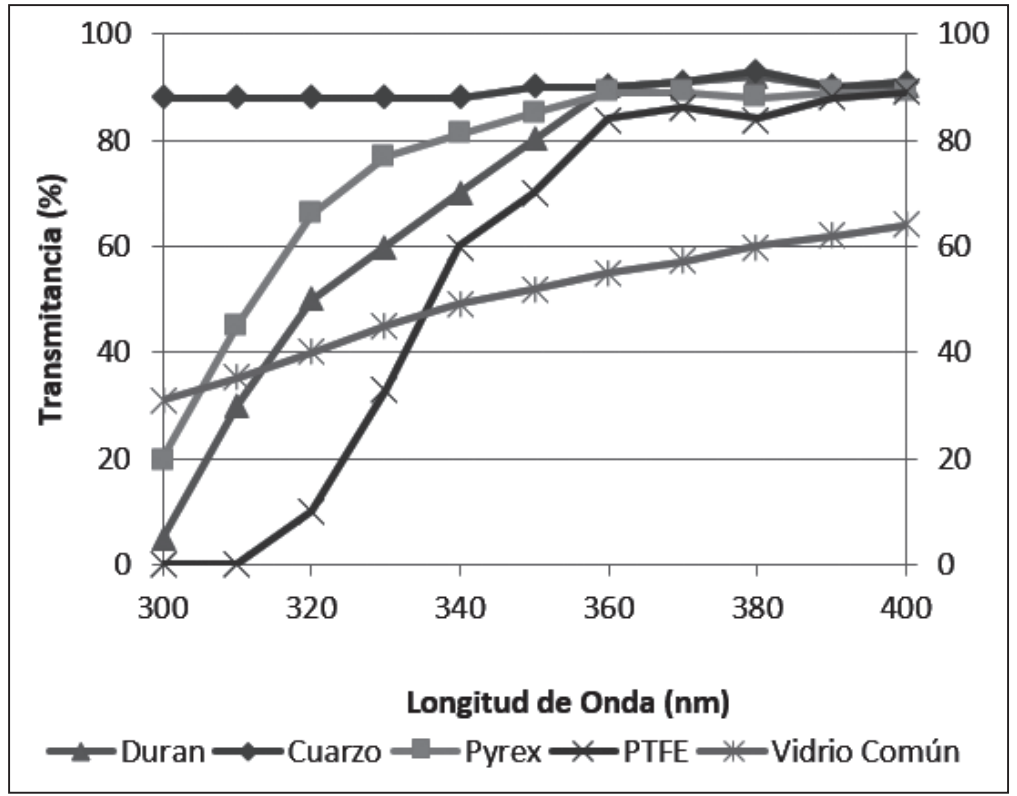

Fig. 3. Diferencia transmitancia entre diferentes tipos de vidrios.

Fuente: elaboración propia. 
En la pasteurización se implantó tubería en PVC de $3 / 4$ " negro para aumentar la temperatura del agua a partir de la absorción de calor.

Estos dos tipos de desinfección solar se situaron sobre una base de aluminio compuesta por cinco tubos de vidrio Pyrex para la fotocatálisis heterogénea y cinco tubos de PVC negros para la pasteurización.

\section{CATALIZADOR}

El catalizador empleado fue Dióxido de Titanio en forma de anatasa $99 \%\left(\mathrm{TiO}_{2}\right)^{2}$, dispuesto a modo de esferas entrelazadas de masilla epoxica (10-15 mm de diámetro) recubiertas con $\mathrm{TiO}_{2}$ como lecho fijo al interior de los tubos de vidrio Pyrex (Fig. 4).
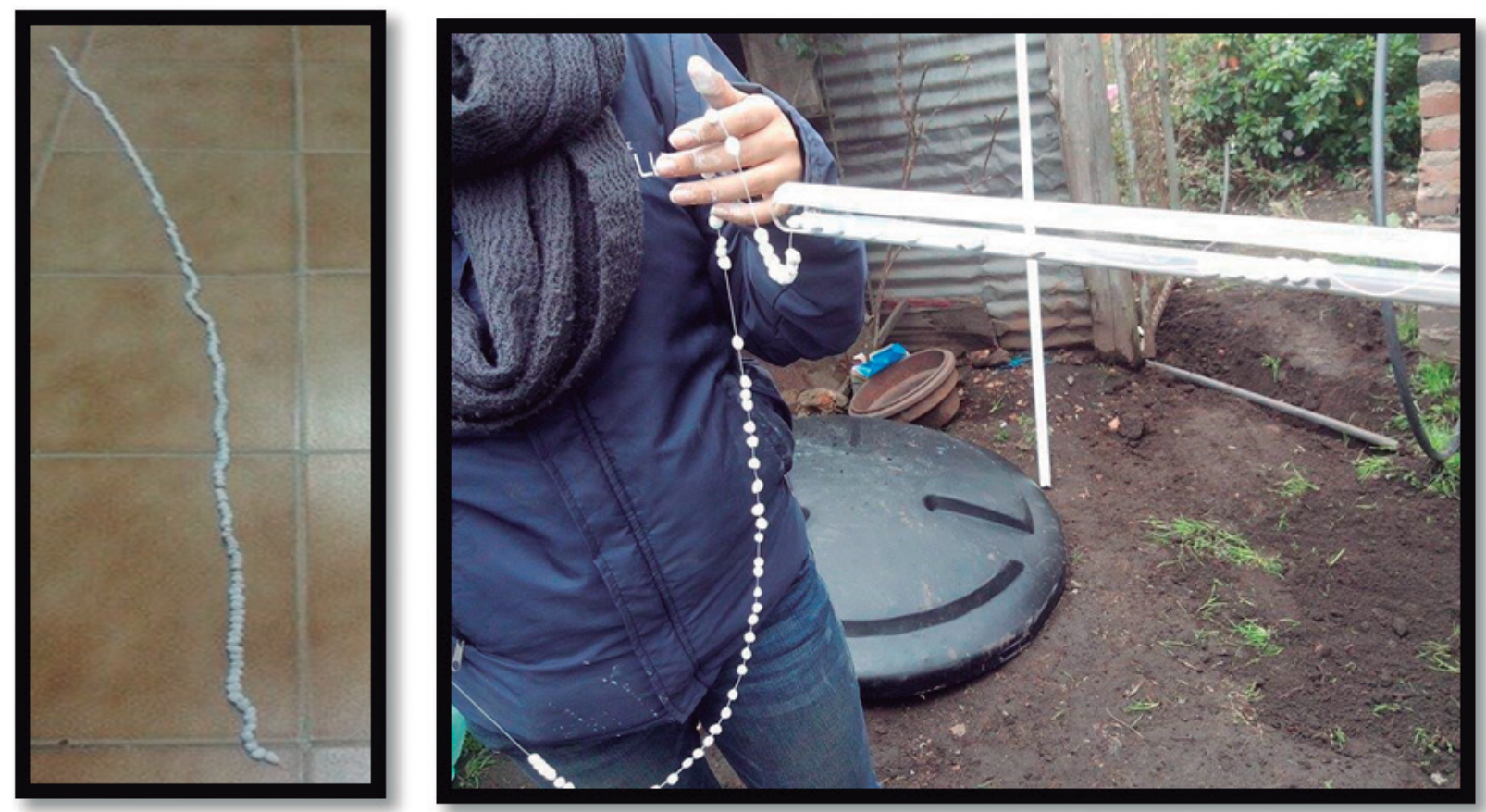

Fig. 4. Implementación de $\mathrm{TiO}_{2}$ en lecho fijo dentro de tubo de vidrio Pyrex

Fuente: elaboración propia.

\section{VARIABLES DE EVALUACIÓN}

Se tomaron muestras concretas en el afluente $y$ en el efluente. Posterior a esto, se hizo un análisis de laboratorio en el Centro Tecnológico de Ambiente y Sostenibilidad de la Universidad de La Salle de características físicas, quí- micas y microbiológicas, teniendo en cuenta las mismas consideraciones del numeral 1.1.

Las principales variables que influyen en la desinfección se presentan en la Tabla 2. De acuerdo con estas, se estableció el número de muestreos y de muestras por realizar.

2 Proveedor del TiO2: MERCK. 
Tabla 2. Número de muestreos

\begin{tabular}{|c|c|c|c|c|c|}
\hline Parametro & $\begin{array}{l}\text { Número de } \\
\text { semanas }\end{array}$ & $\begin{array}{l}\text { Veces por } \\
\text { semana }\end{array}$ & Veces al día & Hora & $\begin{array}{l}\text { Número total } \\
\text { de muestras }\end{array}$ \\
\hline Turbiedad & 4 & 2 & 3 & 08:30 a. m. & \multirow{4}{*}{24} \\
\hline Carga Hidráulica & 4 & 2 & 3 & 11:00 a. m. & \\
\hline Temperatura & 4 & 2 & 3 & \multirow{2}{*}{ 02:00 p. m. } & \\
\hline Índice de radiación & 4 & 2 & 3 & & \\
\hline \multirow{2}{*}{ Coliformes } & \multirow{2}{*}{5} & \multirow{2}{*}{1} & \multirow{2}{*}{2} & 08:30 a. m. & \multirow{2}{*}{10} \\
\hline & & & & $02.00 \mathrm{p} \mathrm{m}$ & \\
\hline
\end{tabular}

Fuente: elaboración propia.

El número total de muestras se determinó teniendo en cuenta la variabilidad climática de la zona.

\section{EVALUACIÓN DEL SISTEMA}

Se comprobó la influencia de las variables que se presentan en la Tabla 3, siguiendo el comportamiento de la turbiedad en la entrada, en la filtración y en la desinfección en términos de la carga hidráulica. Se emplearon dos cargas hidráulicas diferentes durante esta etapa, una correspondiente al caudal máximo de operación, es decir, el flujo de agua que pasa por el sistema cuando la válvula reguladora está abierta en su totalidad, y otra que se encuentra dentro del rango de tasas de filtración recomendadas para filtros lentos $\left(2,4-7,2 \mathrm{~m}^{3} / \mathrm{m}^{2}\right.$.dia) [29].
La presencia o ausencia de coliformes, la temperatura y el índice de radiación también son variables en función de la carga hidráulica. Sin embargo, se estudió el comportamiento de la incidencia de la radiación y la temperatura en la eliminación de microorganismos; asimismo, la influencia de la turbiedad en el paso de la radiación dentro del sistema de fotocatálisis heterogénea.

Las mediciones in situ fueron realizadas para obtener estados del tiempo aleatorios y estudiar la incidencia en la desinfección de acuerdo con el comportamiento de las variables mencionadas. Se verificó el cumplimiento de la resolución 2115 de 2007 de los parámetros señalados en el numeral 1.1. En la Tabla 3 se presentan las mediciones realizadas y los métodos empleados. 
Tabla 3. Parámetros de medición

\begin{tabular}{|l|l|l|l|}
\hline \multicolumn{1}{|c|}{ Parámetros } & \multicolumn{1}{|c|}{ Equipo } & \multicolumn{1}{c|}{ Método } & \multicolumn{1}{c|}{ Descripción } \\
\hline Turbiedad & Turbidimetro & Método nefelométrico & $\begin{array}{l}\text { Método 2130 B Standard } \\
\text { Methods }\end{array}$ \\
\hline Temperatura & Multiparametro & - & $\begin{array}{l}\text { Método 2550 B Standard } \\
\text { Methods }\end{array}$ \\
\hline Coliformes totales & Placas de Petrifilm & Presencia o ausencia & $\begin{array}{l}\text { Certificado de placas de } \\
\text { recuento de coliformes No. } \\
\text { 2006021761 por Invima }\end{array}$ \\
\hline Caudal & $\begin{array}{l}\text { Cronometro y } \\
\text { probeta }\end{array}$ & Volumétrico & - \\
\hline Radiación & Aplicación World UV & $\begin{array}{l}\text { Mide los niveles de } \\
\text { radiación UV }\end{array}$ & $\begin{array}{l}\text { Lanzada por la Asociación } \\
\text { Británica de Dermatólogos }\end{array}$ \\
\hline
\end{tabular}

Fuente: elaboración propia.

Al obtener el índice de radiación se determina la longitud de onda correspondiente (ver Tabla 4).

Tabla 4. Longitud de onda correspondiente a cada índice UV

\begin{tabular}{|l|l|}
\hline \multicolumn{1}{|c|}{ Indice uv } & \multicolumn{1}{|c|}{ Longitud de onda (nm) } \\
\hline $8 \geq 11$ & $400-315$ \\
\hline $03-07$ & $315-280$ \\
\hline $01-02$ & $280-100$ \\
\hline
\end{tabular}

Fuente: elaboración propia.

\section{RESULTADOS Y DISCUSIÓN}

\subsection{Comportamiento de variables de evaluación}

La operación y medición del sistema se realizó aleatoriamente dos días a la semana durante un mes, para obtener diferentes condiciones climáticas y meteorológicas; así, se tomaron ocho mediciones in situ y dos ex situ. Se describe a continuación la evaluación de los parámetros muestreados.

\subsubsection{Turbiedad}

La Fig. 5 presenta las mediciones de turbiedad para dos valores de carga hidráulica, a) $63,36 \mathrm{~m}^{3} \mathrm{~m}^{-2} \operatorname{dia}^{-1}$ y b) $6,9 \mathrm{~m}^{3} \mathrm{~m}^{-2} \mathrm{~d}^{-1}$. 
El comportamiento presentado durante los días seleccionados con carga hidráulica de $63,36 \mathrm{~m}^{3} \mathrm{~m}^{-2} \mathrm{dia}^{-1}$ corresponde a un decrecimiento con valores de turbiedad del $24 \%$, $30 \%$ y $70 \%$ en días cuando las condiciones meteorológicas favorecieron el almacenamiento de agua pluvial por presencia de precipitaciones. No obstante, en días cuando la precipitación fue escasa, el lecho filtrante se saturó y generó arrastre de material y de esta manera aumentó la turbiedad, debido a que hubo bajos volúmenes de agua para operar el sistema, hecho que implicó la intermitencia en el paso del fluido. La Fig. 5 a) muestra que la oferta de lluvia es directamente proporcional a la reducción de la turbiedad. Esta influencia se ve reflejada en el cumplimiento o no del umbral definido por la norma para el parámetro (resolución 2115 de 2007; valor máximo permitido para turbiedad 2 UNT).
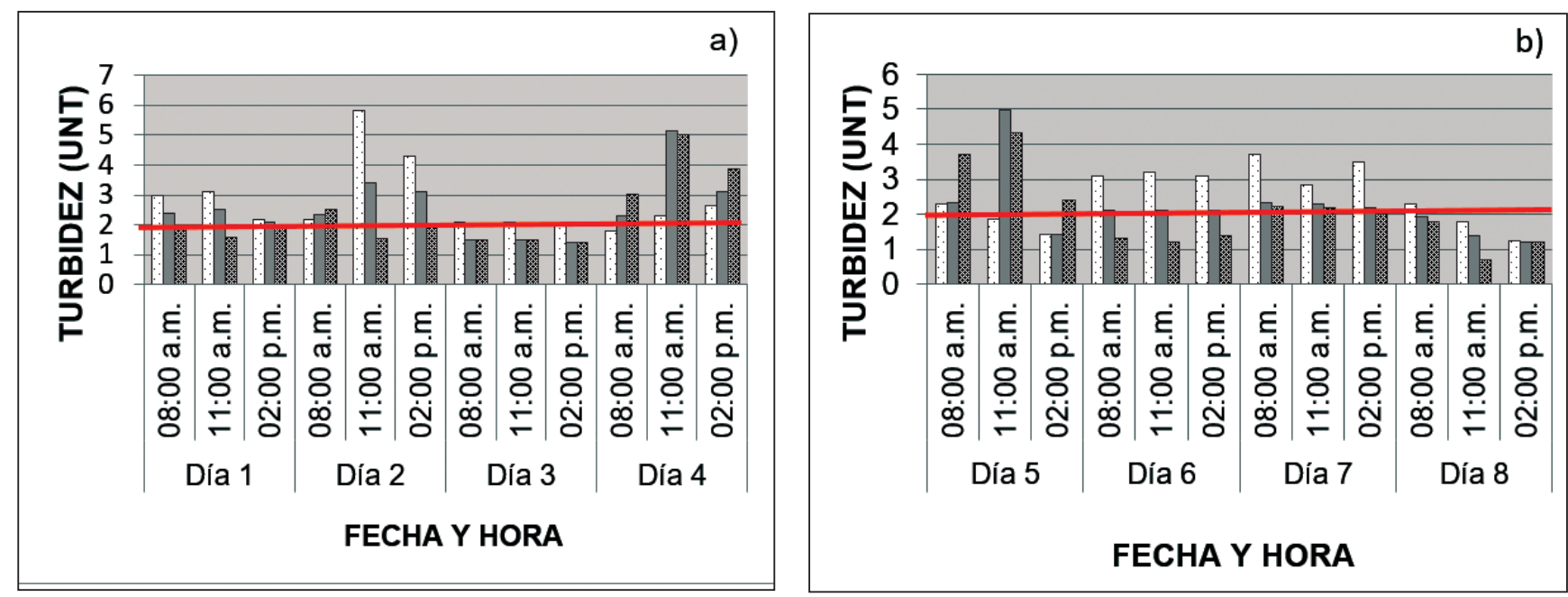

\section{Convenciones \\ $\square$ Inicio sistema \\ Filtración \\ Desinfección solar}

Límite máximo permisible por la resolución 2115 de 2007

Fig. 5. Comportamiento de la turbiedad con una carga hidráulica de a) $63,36 \mathrm{~m}^{3} / \mathrm{m}^{2}-\mathrm{d}$ y b) $6,9 \mathrm{~m}^{3} / \mathrm{m}^{2}-\mathrm{d}$ Fuente: elaboración propia.

La Fig. 5 b) presenta el comportamiento de la turbiedad en el sistema cuando es operado con carga hidráulica $6,9 \mathrm{~m}^{3} / \mathrm{m}^{2}$-d. Se observa una tendencia similar a la de la Fig. 5 a) cuando el sistema funciona en condiciones de bajas precipitaciones. Sin embargo, la mayoría de los días se presentaron reducciones de hasta el $45 \%$, debido a que el flujo del agua obtuvo mayor tiempo de retención. Este comportamiento se relaciona con que la carga hidráulica fue menor (se encontraba dentro del rango de tasas de filtración para 
filtros lentos). Por tanto, operar sistemas de este tipo con cargas hidráulicas bajas ofrece remoción de turbiedad, mejora la eficiencia de la filtración y cumple con la normatividad para agua potable.

\subsubsection{Coliformes}

La Tabla 5 relaciona la presencia o ausencia de coliformes con respecto al índice de radiación y el estado del tiempo al momento del muestreo. Los días que presentaron precipitaciones tuvieron menor incidencia de la radiación y mostraron presencia de coliformes. Bajo entornos nubosos también se observa ausencia de desinfección. Sin embargo, condiciones soleadas y parcialmente soleadas son favorables para la eliminación de microorganismos, con índices de radiación UV entre 4 y 13 o longitudes de onda entre los 280 y $400 \mathrm{~nm}$. No obstante, el estudio de DMA Alrousen presenta la evaluación de un sistema de fotocatálisis heterogénea con $\mathrm{TiO}_{2}$ recubierto en los tubos en donde se evidenció desinfección en condiciones de nubosidad [30].

Los días 9 y 11 muestran eliminación de coliformes a las 8:00 a. m. desde la filtración, lo que manifiesta la posibilidad de eliminar microorganismos desde esta unidad.

Tabla 5. Comportamiento de coliformes en presencia de la radiación

\begin{tabular}{|c|c|c|c|c|c|c|c|c|c|c|}
\hline $\begin{array}{l}\text { Punto de toma de } \\
\text { muestra/hora }\end{array}$ & $\begin{array}{l}\text { 08:00 } \\
\text { a. } \mathrm{m} \text {. }\end{array}$ & $\begin{array}{l}\text { 02:00 } \\
\text { p. } \mathrm{m} \text {. }\end{array}$ & $\begin{array}{l}\text { 08:00 } \\
\text { a. } \mathrm{m} \text {. }\end{array}$ & $\begin{array}{l}\text { 02:00 } \\
\text { p. } \mathrm{m} \text {. }\end{array}$ & $\begin{array}{l}08: 00 \\
\text { a. } \mathrm{m} \text {. }\end{array}$ & $\begin{array}{l}\text { 02:00 } \\
\text { p. } \mathrm{m} .\end{array}$ & $\begin{array}{l}\text { 08:00 } \\
\text { a. } \mathrm{m} \text {. }\end{array}$ & $\begin{array}{l}\text { 02:00 } \\
\text { p. } \mathrm{m} \text {. }\end{array}$ & $\begin{array}{l}08: 00 \\
\text { a. } \mathrm{m} \text {. }\end{array}$ & $\begin{array}{l}\text { 02:00 } \\
\text { p. } \mathrm{m} \text {. }\end{array}$ \\
\hline Día & \multicolumn{2}{|l|}{9} & \multicolumn{2}{|l|}{10} & \multicolumn{2}{|l|}{11} & \multicolumn{2}{|l|}{12} & \multicolumn{2}{|l|}{13} \\
\hline Inicio sistema & $x$ & $x$ & $x$ & $x$ & $x$ & $x$ & $x$ & $x$ & $x$ & $x$ \\
\hline Filtración & $\checkmark$ & $x$ & $x$ & $x$ & $\sqrt{ }$ & $\checkmark$ & $x$ & $x$ & $x$ & $x$ \\
\hline $\begin{array}{l}\text { Desinfección } \\
\text { solar }\end{array}$ & $\sqrt{ }$ & $\sqrt{ }$ & $x$ & $\sqrt{ }$ & $\sqrt{ }$ & $\sqrt{ }$ & $x$ & $x$ & $x$ & $x$ \\
\hline Índice (UV) & 5 & 13 & 2 & 4 & 4 & 11 & 2 & 2 & 2 & 2 \\
\hline Estado del ti & : & 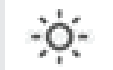 & & & :ơ: & -:ְְ:" & & & فุ! & फ़ं \\
\hline
\end{tabular}

\section{Convenciones}

:-ơ:- Soleado

Lluvioso

Parcialmente soleado

Llovizna

Nublado

$\times$ Presencia de coliformes

$\checkmark$ Ausencia de coliformes

Fuente: elaboración propia. 


\subsubsection{Radiación y la temperatura}

La Fig. 6 presenta los resultados de la eliminación de microorganismos con respecto al índice de radiación y turbiedad para fotocatálisis heterogénea, y temperatura para pasteurización.

La Fig. 6 a) muestra los valores del índice de radiación y de turbiedad con respecto a la desinfección. Los datos de radiación por debajo de 3UV indican en todos los casos presencia de coliformes, con turbiedad entre un rango de 1,2 a 3,0 UNT. Sin embargo, el valor de turbiedad más alto registrado en la desinfección fue de 4 UNT, en presencia de un índice de radiación de $4 \mathrm{UV}$ con eliminación de coliformes. En síntesis, para obtener $100 \%$ de eliminación de microorganismos, debe existir un índice de radiación mayor a 4 UV y turbiedad menor o igual a 4 UNT. Con lo anterior, se muestra que la mayor influencia en la desinfección por fotocatálisis heterogénea se debe a la radiación, desde que se cuente con valores de turbiedad por debajo de 4 UNT.

El Fig. 6 b) presenta los valores de temperatura alcanzados en el sistema de pasteurización, frente a la radiación y la presencia o ausencia de coliformes. De esta manera, se determina si la eliminación de microorganismos se debe a la fotocatálisis o a la pasteurización. La temperatura máxima alcanzada en los días aleatoriamente monitoreados fue de $24,2^{\circ} \mathrm{C}$, con una radiación de $13 \mathrm{UV}$ o 400 $\mathrm{nm}$ y ausencia de coliformes. No obstante, se registró una temperatura mínima de $14,2^{\circ} \mathrm{C}$ con una radiación de 5 U.V. o $300 \mathrm{~nm}$, y eliminación de coliformes en el agua. Esto muestra que al tener una temperatura baja y un índice de radiación por encima de 4 UV se presentó ausencia de coliformes. Si bien, la mayoría de las bacterias mueren a $65^{\circ} \mathrm{C}$ durante 30 minutos $\circ 75^{\circ} \mathrm{C}$ durante 15 minutos [3], dichas temperaturas no fueron alcanzadas y, por tanto, la radiación fue la variable con mayor incidencia en la desinfección.

De igual manera, se ha demostrado que para la desinfección este es un proceso optimo, ya que impide la formación de compuestos halogenados que pueden ser peligrosos y tiene la capacidad de eliminar varios tipos de microorganismos, incluyendo bacterias Gram (+) y (-) [31].

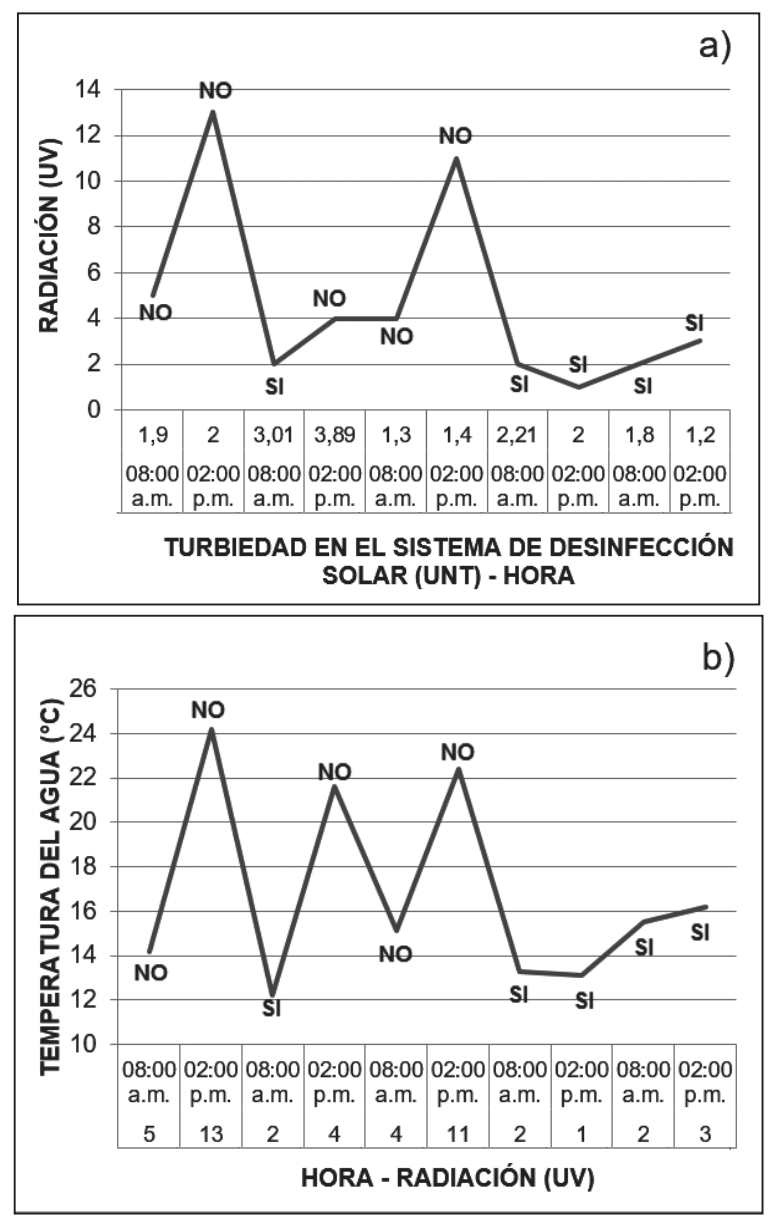

Fig. 6. a) influencia de la radiación y la turbiedad en la desinfección; b) influencia de la radiación y la temperatura en la desinfección: SI: presencia de coliformes, NO: ausencia de coliformes

Fuente: elaboración propia. 


\subsubsection{Caracterización del agua}

La evaluación de los parámetros se hizo con dos cargas hidráulicas diferentes: 63,36 y $6,9 \mathrm{~m}^{3} / \mathrm{m}^{2}-\mathrm{d}$. Los datos de la carga hidráulica $63,36 \mathrm{~m} 3 / \mathrm{m} 2$-dia mostraron que el $70 \%$ de los parámetros medidos cumplió con los valores máximos permisibles, según la resolución 2115 del 2007, mientras que con $6,9 \mathrm{~m}^{3} /$ $\mathrm{m}^{2}$-d, se observa que el $100 \%$ de los parámetros cumple (ver Tabla 6).

Tabla 6. Síntesis de caracterización. IS: inicio del sistema, DS: desinfección solar

\begin{tabular}{|c|c|c|c|c|c|c|c|c|c|}
\hline \multirow{2}{*}{\multicolumn{2}{|c|}{\begin{tabular}{|c|} 
Carga hidráulica \\
$\begin{array}{c}\text { Hora de toma de la } \\
\text { muestra }\end{array}$ \\
\end{tabular}}} & \multicolumn{3}{|c|}{$63,34 \mathrm{~m} 3 / \mathrm{m} 2-\mathrm{d}$} & \multicolumn{3}{|c|}{$6,9 \mathrm{~m}^{3} / \mathrm{m}^{2}-\mathrm{d}$} & \multicolumn{2}{|c|}{$\begin{array}{c}\text { Cumplimiento de la } \\
\text { resolución } 2115 \text { de } \\
2007\end{array}$} \\
\hline & & $\begin{array}{l}08: 00 \\
\text { a. } \mathrm{m} \text {. }\end{array}$ & $\begin{array}{l}\text { 11:00 } \\
\text { a. } m \text {. }\end{array}$ & $\begin{array}{l}\text { 02:00 } \\
\text { p. m. }\end{array}$ & $\begin{array}{l}08: 00 \\
\text { a. } \mathrm{m} .\end{array}$ & $\begin{array}{l}11: 00 \\
\text { a. } \mathrm{m} \text {. }\end{array}$ & $\begin{array}{l}\text { 02:00 } \\
\text { p. m. }\end{array}$ & Carga 1 & Carga 2 \\
\hline \multirow{2}{*}{$\mathrm{pH}$} & IS & 5,85 & 6,32 & 6,54 & 5,9 & 5,63 & 5,76 & - & - \\
\hline & DS & 5,61 & 6,24 & 6,73 & 5,4 & 5,52 & 5,7 & - & - \\
\hline \multirow{2}{*}{ Conductividad } & IS & 0,08 & 0,05 & 0,01 & 0,04 & 0,01 & 0,01 & - & - \\
\hline & DS & 0,02 & 0,05 & 0,04 & 0,02 & 0,01 & 0,01 & - & - \\
\hline \multirow{2}{*}{ SD } & IS & 0,04 & 0,02 & 0,02 & 0,02 & 0,01 & 0,01 & - & - \\
\hline & DS & 0,01 & 0,03 & 0,02 & 0,01 & 0,01 & 0 & - & - \\
\hline \multirow{2}{*}{ Temperatura } & IS & 11,1 & 12,6 & 15,9 & 10,8 & 12,3 & 12,1 & - & - \\
\hline & DS & 12,2 & 21,8 & 21,6 & 15,5 & 12,3 & 16,2 & - & - \\
\hline \multirow{2}{*}{ Turbidez } & IS & 1,78 & 2,3 & 2,62 & 2,3 & 1,8 & 1,25 & $\begin{array}{l}\text { No } \\
\text { cumple }\end{array}$ & $\begin{array}{l}\text { Sí } \\
\text { cumple }\end{array}$ \\
\hline & DS & 3,01 & 5,02 & 3,89 & 1,8 & 0,69 & 1,2 & $\begin{array}{l}\text { Sí } \\
\text { cumple }\end{array}$ & $\begin{array}{l}\text { Sí } \\
\text { cumple }\end{array}$ \\
\hline \multirow{2}{*}{$\begin{array}{l}\text { Presencia de } \\
\text { Coliformes }\end{array}$} & IS & SI & - & SI & SI & - & SI & $\begin{array}{l}\text { No } \\
\text { cumple }\end{array}$ & $\begin{array}{l}\text { No } \\
\text { cumple }\end{array}$ \\
\hline & DS & $\mathrm{SI}$ & - & NO & SI & - & $\mathrm{SI}$ & $\begin{array}{l}\text { Sí } \\
\text { cumple }\end{array}$ & $\begin{array}{l}\text { No } \\
\text { cumple }\end{array}$ \\
\hline \multirow{2}{*}{ Dureza } & IS & 0,044 & 0,52 & 0,56 & 0,22 & 0,18 & 0,12 & $\begin{array}{l}\text { Sí } \\
\text { cumple }\end{array}$ & $\begin{array}{l}\text { Sí } \\
\text { cumple }\end{array}$ \\
\hline & DS & 0,064 & 0,64 & 0,6 & 0,18 & 0,1 & 0,32 & $\begin{array}{l}\text { Sí } \\
\text { cumple }\end{array}$ & $\begin{array}{l}\text { Sí } \\
\text { cumple }\end{array}$ \\
\hline \multirow{2}{*}{ Aluminio } & IS & 0 & 0 & 0 & 0 & 0 & 0 & $\begin{array}{l}\text { Sí } \\
\text { cumple }\end{array}$ & $\begin{array}{l}\text { Sí } \\
\text { cumple }\end{array}$ \\
\hline & DS & 0 & 0 & 0 & 0 & 0 & 0 & $\begin{array}{l}\text { Sí } \\
\text { cumple }\end{array}$ & $\begin{array}{l}\text { Sí } \\
\text { cumple }\end{array}$ \\
\hline
\end{tabular}

Fuente: elaboración propia. 


\section{CONCLUSIONES}

La implementación de un sistema de tratamiento constituido por filtración y desinfección solar por fotocatálisis heterogénea y pasteurización, para un afluente producto de la captura de aguas lluvias, pudo contribuir a la carencia del recurso en una locación vulnerable del sur de Bogotá; no obstante, si se presenta el hecho de que en la localidad en la que se desea implementar el piloto no se cuenta con probabilidad alta de precipitación, se puede buscar una fuente de agua con bajos niveles de turbiedad para tratar, como se ha mencionado anteriormente; el sistema de tratamiento de fotocatálisis solo es efectivo si la turbiedad del agua es baja ya que, si fuera al contrario, los rayos ultravioleta no pueden actuar efectivamente con el catalizador dióxido de titanio.

Dadas las condiciones meteorológicas y climatológicas de la zona donde se implementó el proyecto, las variables más importantes fueron la carga hidráulica en función de la radiación, la turbiedad, la presencia de coliformes y la temperatura, variables que determinaron las unidades de captación de agua lluvia, tratamiento de filtración y desinfección solar, empleando materiales adecuados para cada una de las variables: carga hidráulica y turbiedad (Filtro lento de arena), radiación (vidrio Pyrex y base de aluminio), temperatura: (tubo negro y tapa en acrílico), coliformes (sistema de desinfección solar por fotocatálisis heterogénea con catalizador dióxido de titanio y pasteurización).

El proceso de análisis permitió evidenciar que en la filtración, a mayor carga hidráulica, menor eficiencia en cuanto a remoción de turbiedad, factor que influye en el paso de la radiación. Asimismo, en los resultados de la influencia de la radiación y la turbiedad en la desinfección (presencia de coliformes), se observó que con índices de radiación que van desde los 4UV se presenta desinfección en el sistema de fotocatálisis heterogénea.

La evaluación del sistema de desinfección solar indica que no hubo ausencia de coliformes por la pasteurización, puesto que la temperatura ambiente de la zona no fue suficiente para elevar la temperatura del agua en el sistema, lo que indica que la desinfección se logró por la fotocatálisis heterogénea. Lo anterior basado en que este proceso causa daño celular por la generación de radicales $\mathrm{OH}$ en la superficie del fotocatalizador y por la absorción directa de la radiación UV por las células, lo que contribuye en gran medida a promover la inactivación celular.

\section{AGRADECIMIENTOS}

Gracias a Viviana Carvajal, por su colaboración en la construcción del proyecto; de igual manera a Liced Melo, Jairo Sarmiento, Eladio Guerrero y Fanny Guerrero, por el apoyo. También agradecemos el acompañamiento y la asesoría continua del ingeniero Julio $\mathrm{Ce}$ sar Ramírez.

\section{REFERENCIAS}

[1] L. De Vargas. Manual I: Tratamiento de agua para consumo humano, plantas de filtración rápida. Lima, Perú: Centro Panamericano de Ingeniería Sanitaria y Ciencias del Ambiente [CEPIS], Organización panamericana de la Salud [OPS], 2004. 
[2] L. D. Sánchez y E. Y. Caicedo, "Uso del agua lluvia en la Bocana Buenaventura," en Conf. Int. Usos Múltiples del Agua: para la Vida y el Desarrollo Sostenible, Cali, Colombia, 2003.

[3] F. Solsona y J. P. Méndez. Desinfección del agua. Lima, Perú: Centro Panamericano de Ingeniería Sanitaria y Ciencias del Ambiente [CEPIS], Organización panamericana de la Salud [OPS], 2002.

[4] G. Sauthier, "Preparación y caracterización de capas delgadas y estructuras de óxido de titanio para aplicaciones fotovoltaicas activadas mediante radiación UV o visible," Tesis de doctorado, Facultad de Ciencias, Departamento de Química, Universidad Autónoma de Barcelona, Barcelona, 2012.

[5] K. D. Caraballo, "Diseño, construcción y evaluación de un reactor fotocatalítica solar de placa plana para degradar ácido tereftálico," Tesis de pregrado, Facultad de Ingeniería, Universidad de Cartagena, Cartagena, 2012.

[6] L.F.Garcés, E. A. Mejíay J.J.Santamaría, "La fotocatálisis como alternativa para el tratamiento de aguas residuales," Revista Lasallista de investigación, vol. 1, no. 1, pp. 83-92, 2004.

[7] C. A. Castro, A. L. Arámbula, A. Centeno y S. A. Giraldo, "Degradación Heliofotocatalítica de Escherichia coli en Sistemas tipo Desinfección SODIS, con Dióxido de Titanio Modificado," Información Tecnológica, vol. 20 , no. 6 , pp. 29-36, 2009. DOI: https://doi.org/10.4067/s071807642009000600005

[8] J. A. Arroyavey L. F. Garcés, “Evaluación de la degradación del pesticida Mertect empleando procesos avanzados de oxidación (PAO)," Revista Producción + Limpia, vol. 6, no. 2, pp. 9-18, 2007.

[9] J. A. Arroyave, L. F. Garcés y A. F. Cruz, "Fotodegradación de las aguas residuales con pesticida Mertect en la industria bananera empleando Fotocatálisis con Dióxido de Titanio y Lámpara de Luz Ultravioleta," Revista Lasallista de Investigación, vol. 4, no. 1, pp. 8-9, 2007.

[10] J. Blanco, S. Malato, C. A. Estrada, E. R. Bandala et al. "Purificación de Aguas por Fotocatálisis Heterogénea: Estado del Arte. Parte 1," 2014. [En línea]. Disponible en: http://www.estrucplan. com.ar/Producciones/entrega. asp? IdEntrega=2958.

[11] F. F. Garcés, M. L. Hernández, G. A. Peñuela, A. Rodríguez y J. A. Salazar, "Degradación de aguas residuales de la industria textil pormedio defotocatálisis," Revista Lasallista de investigación, vol. 2, no. 1, pp. 15-18, 2005.

[12] I. Restrepo, L. D. Sánchez, A. Galvis, J. Rojas e I. J. Sanabria. Avances en investigación y desarrollo en agua y saneamiento. Cali, Colombia: Editorial Primera, Universidad del Valle, 2007.

[13] C. Gutiérrez, L. Robles, F. Ortiz y L. Martínez, "Desinfección foto-catalítica del agua para consumo humano usando luz solar y dióxido de titanio $\left(\mathrm{TiO}_{2}\right)$ 
inmovilizado, 2004. [En línea]. Disponible en: http://www.elaguapotable.com/ DESINFECCI\%C3\%93N\%20FOTOCATAL \% C3\% 8DTICA \% 20DEL \% 20 AGUA\%20PARA $\% 20$ CONSUMO $\% 20$ HUMANO $\% 20$ USANDO $\% 20$ LUZ $\% 20$ SOLAR\%20Y\%20DI\%C3\%93XIDO\%20 DE \% 20 TITANIO\% 20 (TiO2)\% 20 INMOVILIZADO.pdf.

[14] M. Iborra, J. Tejero y F. Cunill, "Apuntes: Reactores Multifásicos," 2013. [Enlínea]. Disponible en: http://diposit.ub.edu/ dspace/bitstream/2445/33262/1/ APUNTES\%20RM.pdf

[15] P. Fernández, “Desinfección con reactores solares: experiencia operativa," 2005. [En línea]. Disponible en: https://www. psa.es/es/projects/solarsafewater/ documents/libro/16_Capitulo_16.pdf.

[16] S. Malato, J. Blanco, D. Alarcón, M. Maldonado y P. Fernández, "Photocatalytic decontamination and disinfection of water with solar collectors," Catalysis Today, vol. 122, 2007, pp. 137-149. DOI: https://doi.org/10.1016/j. cattod.2007.01.034

[17] G. M. Restrepo, L. A. Ríos, J. M. Marín, J. F. Montoya y J. A. Velázquez, "Evaluación del tratamiento fotocatalitico de aguas residuales industriales empleando energía solar," Dyna, vol. 75, no. 155, pp. 145-153, 2008.

[18] A. Rincón, S. Giraldo y C. Pulgarín, "Desinfección de agua por fotocatálisis. Aspectos básicos," 2005. [En línea] Disponible en: https://www.psa.es/es/ projects/solarsafewater/documents/ libro/13_Capitulo_13.pdf
[19] J. J. Hermosillo, "Agua potable para microcomunidades con energía solar," Renglones: Revista del ITESO, vol. 1, no. 49, pp. 85-91, 2001.

[20] R. Meierhofe y M. Wegelin, "Guía de aplicación: Desinfección solar del agua," 2003. [En línea]. Disponible en: http://www.sodis.ch/methode/anwendung/ausbildungsmaterial/dokumente_material/manual_s.pdf.

[21] J. H. Castillo, A. López y E. R. Bandala, "Desinfección de agua mediante el uso de tecnologías emergentes basadas en procesos avanzados de oxidación," Revista Temas selectos de Ingeniería de alimentos, vol. 4, no. 1, pp. 74-83, 2010.

[22] $3 \mathrm{M}$, "Placas $3 \mathrm{M}^{\mathrm{TM}}$ Petrifilm ${ }^{\mathrm{TM}}$ E. coli/ Coliformes," 2016. [En línea]. Disponible en: http://solutions.productos3m.es/ wps/portal/3M/es_ES/FoodSafetyEU/ FoodSafety/ProductInformation/ ProductCatalogue/?PC_Z7_RJH9U5 2300DK40IMRSPA7P2065000000_ nid=COWJ62882Vbe8SD7TQV1 GLgl

[23] J. M. Preciado, Z. R. Mejia y J. M. Arganis, "Análisis y aplicación de métodos temporales para completar registros faltantes de lluvia en la cuenca del rio Bolaños," en $V$ Congr. Int. De meteorología de la OMMAC, Acapulco, México, 2011.

[24] Organización panamericana de la Salud [OPS], Centro Panamericano de Ingeniería Sanitaria y Ciencias del Ambiente [CEPIS], "Guía de diseño para captación del agua lluvia," 2004. [En línea]. Disponible en: http://www.bvsde. ops-oms.org/bvsacd/cd47/lluvia.pdf 
[25] R. Riera, “Filtro Arena 20”,36” y 48," 2006. [En línea]. Disponible en: https://www. uclm.es/area/ing_rural/Catalogos/ HidraulicaRiegos/RegaberFiltroArena. pdf

[26] C. Torres, “Procedimiento para la prueba de aforo volumétrico," 2006. [En línea]. Disponible en: http://www. utp.ac.pa/documentos/2011/pdf/ PCUTP-CIHH-AH-103-2006.pdf

[27] Y. L. Ramírez, "Diseño, construcción y puesta en marcha de un reactor tubular fotocatalítica (UV-A) para la degradación de desechos químicos orgánicos," Tesis de pregrado, Facultad de Tecnologías, Universidad tecnológica de Pereira, Pereira, 2013.

[28] S. Malato, J. Blanco, R. Maldonado, P. Fernández et a.l., "Engineering of solar photocatalytic collectors," Solar Energy, vol. 77, no. 5, 2004, pp.513-524.
DOI: https://doi.org/10.1016/j.solener.2004.03.020

[29] J. A. Romero. Purificación del Agua. Bogotá, Colombia: Escuela Colombiana de Ingeniería, 2006.

[30] D. Alrousan, M. López, P. Dunlop y P. Fernández, "Solar photocatalytic disinfection of water with immobilised titanium dioxide in re-circulating flow CPC reactors," Applied Catalysis B: Environmental, vol. 128, pp. 126-134, 2012. DOI: https://doi.org/10.1016/j. apcatb.2012.07.038

[31] G. X. Velásquez, "Remoción de coliformes fecales en aguas residuales generadas en una granja avícola, utilizando fotocatálisis heterogénea como una alternativa," Tesis de pregrado, Facultad de Ingeniería Química, Universidad de San Carlos de Guatemala, Guatemala, 2016. 